\title{
CRYOGENIC TEMPERATURE MEASUREMENT FOR LARGE APPLICATIONS
}

\author{
J. Ylöstalo (1,4), P. Berglund (1,4), J. Kyynäräinen (1,4), \\ T.O. Niinikoski (2), and R. Voutilainen (3)
}

\begin{abstract}
We have developed a resistance thermometry system for the acquisition, control and monitoring of temperature in large-scale cryogenic applications. The resistance of the sensor is converted to a voltage using a self-balancing AC bridge circuit featuring square-wave excitation currents down to $1 \mathrm{nA}$. The system is easily scalable and includes intelligent features to treat special situations such as magnet quenches differently from normal operation.
\end{abstract}

Submitted to Cryogenics

(1) Helsinki University of Technology, Low Temperature Laboratory, 02150 Espoo, Finland

(2) CERN, CH-1211 Geneva 23, Switzerland.

(3) RV-Elektroniikka Oy Picowatt, 01510 Vantaa, Finland.

(4) Helsinki University of Technology, Institute of Particle Physics Technology, 02150 Espoo, Finland. 


\section{INTRODUCTION}

\section{Challenge of the future}

In the near future several large cryogenic facilities will be built for the research of high energy physics and fusion energy. These facilities use massive superconducting magnets, whose control systems need the measurement of temperature in many points. In the CERN Large Hadron Collider [1], for example, there will be 1232 twin-bore dipole magnets of $14.2 \mathrm{~m}$ length and $8.4 \mathrm{~T}$ strength operated at $1.9 \mathrm{~K}$ temperature, and hundreds of other types of magnets operated at $1.9 \mathrm{~K}$ or $4.5 \mathrm{~K}$. The temperature data need to be recorded to control the magnet cooldown or warm-up, and to monitor the cryogenic system during operation.

These systems must often operate very close to their limits so that a rather small increase in temperature may cause a quench. This requires good stability of temperature and therefore fine resolution in its monitoring. In LHC [1], for example, the superfluid helium coolant will be heat sinked at $1.8 \mathrm{~K}$, only $0.1 \mathrm{~K}$ below the projected temperature of the magnet coils. At the maximum energy and ultimate beam intensity the coils may operate only $0.05 \mathrm{~K}$ below their quench limit temperature.

\section{Special requirements}

Typically, the temperature measurement instruments for superconducting applications should thus be:

1) Specified for cryogenic temperatures;

2) Reliable and accurate;

3) Fast in response, and capable of time stamping the data with a good time resolution, so that it can be related to dynamic events (such as quenches) in other parts of the control system;

4) Remotely controllable and intelligent, in order to make it possible to operate a large system.

5) Scalable and affordable so that large series can be procured and maintained in a costefficient manner.

Presently no single instrument fulfils all these requirements. The existing industrial devices have good time resolution but lack features important for cryogenic applications. The precise and extremely versatile low temperature thermometry instruments for small laboratory systems are expensive and also rather slow as the measuring power in the cryogenic sensor has to be kept low. Thermal contact and thermal conductivity determine how much power can be dissipated in the sensor and together with the heat capacity of the materials they give the thermal time constants seen in the measuring process.

We have therefore decided to specify and develop a dedicated instrumentation system for large scale cryogenic thermometry, where cost and scalability are important factors in addition to the specifications in accuracy, reliability and intelligent features. 


\section{Outline of the paper}

The first item of the above list will be discussed in Section 2, with particular view on the superconducting applications above $1.8 \mathrm{~K}$ temperature. Reliability and accuracy (item 2) are required for fault detection and alarm generation, and they involve the control of electromagnetic interference (EMI) and optimisation of noise performance, in addition to the elimination of systematic problems related to the cryogenic environment. Reliability also depends on the circuit design principles and on the choice of components; use of radiation hard components, for example, will strongly influence cost. The control of EMI and the optimisation of noise will be discussed in Section 3 together with the system specifications and circuit design, and brief notes on the remaining subjects can be found throughout our paper.

During quench testing the instruments must be capable of recording the temperature transients with fine resolution both in time and in temperature. This is also vitally important for recording the transients due to accidental quenches interrupting normal operation, because such transient data will greatly help finding and eliminating the cause of the event. Based on typical sensor and component response times which are discussed in Section 2, we have chosen the sampling rate of $10 \mathrm{~Hz}$ and the time stamp resolution of $10 \mathrm{~ms}$, conforming to standards in industrial control systems.

Item 4 will be discussed in Section 4, together with communication with control systems, and with the user interface which was designed to satisfy the needs of the pilot application. Scalability (item 5) is based on the system concept which will allow to build tree-like data acquisition and reduction systems with functional approach in architecture [2]. This will be briefly treated in Sections 3 and 4. The experimental performance is compared with the design values in Section 5. Projections for improving and extending the system are discussed in the concluding Section 6.

Sensor design and questions related with their use in radiation and magnetic fields and other hostile environments are important in the future large applications. As the choice of sensors influences the optimisation of their readout, we shall begin by briefly reviewing the cryogenic resistance thermometry in Section 2 with references to the wide literature on the topic.

\section{CRYOGENIC ENVIRONMENT}

Even if several methods for measuring the temperature are possible, resistance thermometry is most easily applied for measuring the temperature of cryogenic components. Our instrument is primarily designed for this purpose. This does not preclude that the same instrument with minor modification can be used as a diode thermometer or even for thermometry using an array of tunnel junctions (micro miniature field-independent sensors).

\section{Resistance thermometry}

Resistance thermometry [3] allows robust, reliable and fast measurement of temperature in cryogenic applications using calibrated secondary sensors, and it is also used for the interpolation between the primary ITS-90 scale of fixed points [4]. In comparison with thermometry based on the measurement of vapour pressure, thermocouple voltage, capacitance or diode voltage for example, the resistance thermometry has advantages in one or more of the following: low sensor cost, fast response, high sensitivity and small size. Resistance sensors are therefore the most widely used ones in cryogenics. Many excellent review articles [5, 6, 7, 
$8,9]$ treat cryogenic resistance thermometry, and we shall only briefly outline the most popular sensor types below.

The main resistance sensor types include metallic, metal oxide and semiconductor elements, in the geometry of bulk, wire or thin film. The most important among the metallic sensors are made of pure platinum (Pt); such sensors are used for the interpolation between the fixed points of the primary scale. Other metallic sensors are made of pure metals or alloys such as rhodiumiron (RhFe), nickel, nickel-iron, copper etc. Metallic sensors, in particular thin-film RhFe, are relatively immune to radiation but suffer moderately from magnetoresistive effects which must be corrected for if absolute accuracy is desired in magnetic field.

The semiconductor sensors include doped germanium (Ge) and silicon ( $\mathrm{Si}$ ), and carbon in thin films or bulk composite form. The Ge sensors may be doped by melt processing or by nuclear transmutation, and the Si sensors are processed onto the surface of pure Si wafers by implanting or diffusing suitable impurities. Ge sensors can also be vacuum deposited onto sapphire, for example. The Ge and Si sensors, which can be tailored to yield a very good sensitivity in a rather wide temperature range, suffer from substantial magnetic field effects and from sensitivity to radiation damage. The carbon composite sensors are ordinary radio resistors usually chosen for their characteristics and stability, but some of them have also been tailored for the present application. These and the carbon glass sensors offer moderately small corrections for magnetic field effects.

The ruthenium oxide $(\mathrm{RuO})$ thick-film sensor has a somewhat limited sensitivity but covers a very large range of temperatures and offers the best performance in high fields. Owing to its principle, it is also relatively immune to radiation damage.

The desired sensitivity and accuracy of the measurement of temperature in large superconducting applications depends on the definition of the safe operation conditions. Supposing that the quench limit is defined at $50 \mathrm{mK}$ above the coil temperature, we assume that the temperature in the range of $1.8 \ldots 4.5 \mathrm{~K}$ must be monitored with an accuracy of at least 5 $\mathrm{mK}$ and with a resolution of about $1 \mathrm{mK}$. The $\mathrm{Ge}, \mathrm{RuO}$, carbon composite and carbon glass sensors have typical logarithmic sensitivities of $1 \ldots 4$ in this range, whereas $\mathrm{Pt}$ and $\mathrm{RhFe}$ sensors are limited to $0.16 \ldots 0.26$. The former require to measure typical resistance values around $1 \mathrm{k} \Omega$ with a resolution of $0.02 \%$ and absolute accuracy of $0.1 \%$, whereas the latter call for the measurement of $1 \ldots 2 \Omega$ resistances with $0.005 \%$ and $0.05 \%$ resolution and accuracy, respectively. In our design we have aimed at meeting the specification with $\mathrm{Ge}, \mathrm{RuO}$, carbon composite and carbon glass sensors at $1.8 \ldots 4.5 \mathrm{~K}$ temperatures, and note that similar specifications can be obtained above $20 \mathrm{~K}$ using Pt and RhFe sensors.

The response of the resistance sensor can be tailored and it may be faster than $1 \mathrm{~ms}$ for miniature units around $1 \mathrm{~K}$ [10]. The thermal response of the object whose temperature is under monitoring, however, is often limited by the thermal diffusivity of materials and by geometric design. These considerations lead us to propose the rate of $10 \mathrm{~Hz}$ for monitoring the temperature, rather than the more usual rate of $100 \mathrm{~Hz}$ in industrial control systems. The time stamp resolution, however, was maintained at $10 \mathrm{~ms}$. In order to monitor faster thermal phenomena, such as second sound in superfluid ${ }^{4} \mathrm{He}$, bolometric techniques must be used rather than our instrument which emphasises accurate thermometry.

The sources of systematic errors in cryogenic resistance thermometry include self-heating of the sensor, heating due to conduction through leads and to EMI pick-up, thermal voltages in leads (in DC measurement), variation of lead resistance (in 2- and 3-lead measurements), and 
errors due to preamplifier bias current. Without going into the details of these considerations, we note that most of them can be eliminated by using balanced 4-wire AC bridge techniques. The remainder, i.e. heating due to conduction through leads and of EMI pick-up, require the use of low-conductivity cryogenic lead materials which have a sizeable resistance and which are appropriately heat sinked, and the placement of RF filters to all leads at the point of entrance to the cryogenic vacuum vessel (we exclude in large-scale applications the use of Faraday cage techniques). The bridge circuit must therefore be able to operate with resistive leads and with parasitic reactances in the filters.

Based on the above considerations we have chosen to design a self-balancing active AC bridge circuit with four measurement ranges covering resistances from 0 to $200 \Omega, 2 \mathrm{k} \Omega, 20$ $\mathrm{k} \Omega$ and $200 \mathrm{k} \Omega$, and the choice of three levels of constant current excitation in steps of one decade. These resistance ranges provide coverage for all sensors in their useful temperature domain, and the circuit can be close to optimum in most of the resistance domain. The three excitations allow to test self-heating below $10 \mathrm{~K}$, and the lowest one extends the temperature range to well below $100 \mathrm{mK}$.

\section{SYSTEM CONCEPT AND CIRCUIT DESIGN}

\subsection{Conceptual design of the measurement system}

Our aim was thus to construct a prototype data acquisition system for measurement, control and monitoring of cryogenic temperature which is specifically designed for large-scale systems. The measurement is based on the self-balancing active AC resistance bridge technique developed by Picowatt, which features low power dissipation (below $1 \mathrm{pW}$ at lowest excitation) and immunity against electromagnetic interference and thermal voltages. This technique allows to measure accurately very low temperatures using the resistance sensors which were briefly described in the previous Section.

The conceptual design of the system includes in addition the following:

- One shielded cable links the RF-shielded enclosure of the circuitry to the hermetic receptacle of the cryogenic vacuum vessel. This provides good control of EMI in the most sensitive part, and allows to use one multiway RF filter at the inlet to the cryogenic vacuum. The length of the cable may be several metres in order to locate the circuitry in an environment compatible with the requirements of ambient temperature, radiation and magnetic field.

- The RF-shielded enclosure contains up to 8 resistance bridge cards and one processor, which acquires and reduces data from the bridge cards.

- The processor communicates with host computers using the TCP/IP protocol and Ethernet port which may be optoisolated so that there are no other galvanic links to the circuitry than the mains power feed and the sensor cable.

- The mains power feed is RF-filtered and a high-grade toroidal transformer is used.

- The processor performs a series of intelligent tasks which can be defined from the user interface. 
These principles have proved the Faraday cage techniques unnecessary even in ultralow temperature resistance thermometry below $10 \mathrm{mK}$, in applications where other instrument connections do not require such techniques.

\subsection{AC bridge circuit design}

Figure 1 shows the block diagram of the bridge circuit. The frequency of the square-wave AC excitation signals is $12.5 \mathrm{~Hz}$ and it is derived from the $50 \mathrm{~Hz}$ mains power wave shape. The subharmonic relation with the mains frequency eliminates beats or offset due to accidental pickup of spurious signals related with the power network.

The prototype unit is capable of measuring resistances in four ranges of $200 \Omega$ to $200 \mathrm{k} \Omega$ (with about $25 \%$ overranging capacity), and has in each range three excitation current amplitudes which give midrange ( $100 \Omega$ to $100 \mathrm{k} \Omega$ ) voltages over the sensor of $100 \mu \mathrm{V}, 1 \mathrm{mV}$ and $10 \mathrm{mV}$. The highest excitation gives a stable low noise readout and is recommended at temperatures above $4 \mathrm{~K}$, while the lowest one is usable well below $100 \mathrm{mK}$ e.g. in dilution refrigerators.

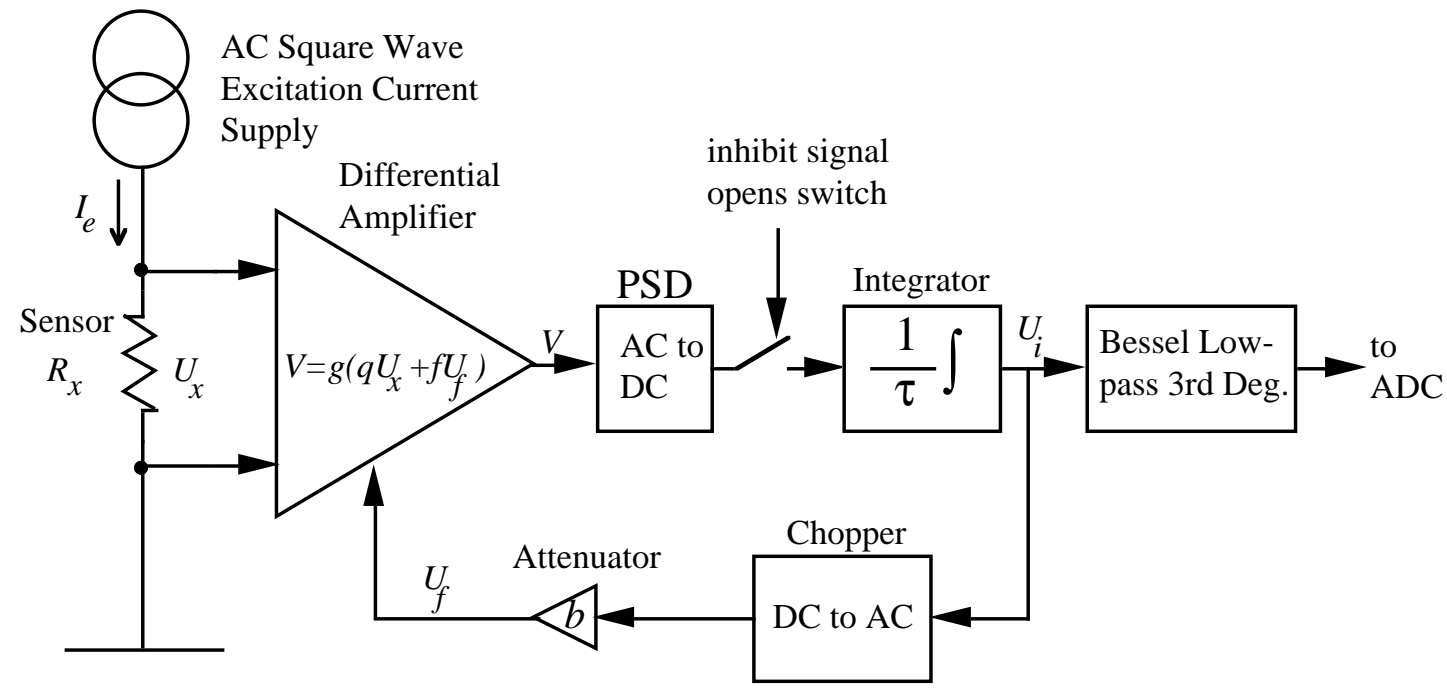

Figure 1: Block diagram of the self-balancing active AC resistance bridge circuit. The sensor resistor is connected with four wires to the bridge, thus eliminating errors due to the lead resistance. The phase sensitive detector (PSD) is followed by a gate which inhibits the propagation of the voltage transients, due to the inversion of the polarity of the excitation and to the reactance of the measurement leads, into the integrator.

Using the notation of the block diagram presented in Figure 1 and the parameters

$$
\begin{array}{ll}
a=f \cdot b \cdot g & =\text { feedback gain; } \\
p=q \cdot g & =\text { signal gain before integrator; } \\
\tau & =\text { integrator time constant }
\end{array}
$$

the amplitude and impulse response functions of the circuit can be written in the form

$$
\begin{gathered}
|H(j \omega)|=\frac{p}{\sqrt{a^{2}+(\omega \tau)^{2}}} ; \\
h(t)=\frac{p}{\tau} e^{a t / \tau} .
\end{gathered}
$$


We note that we may control the DC gain $p / a$ and response time constant $\tau / a$ by controlling the various amplifier gains. This is used for optimising the dynamic range and the response bandwidth under the various conditions of excitation current and resistance range by selecting the appropriate gains in the amplifiers.

\subsection{Noise model for the bridge circuit}

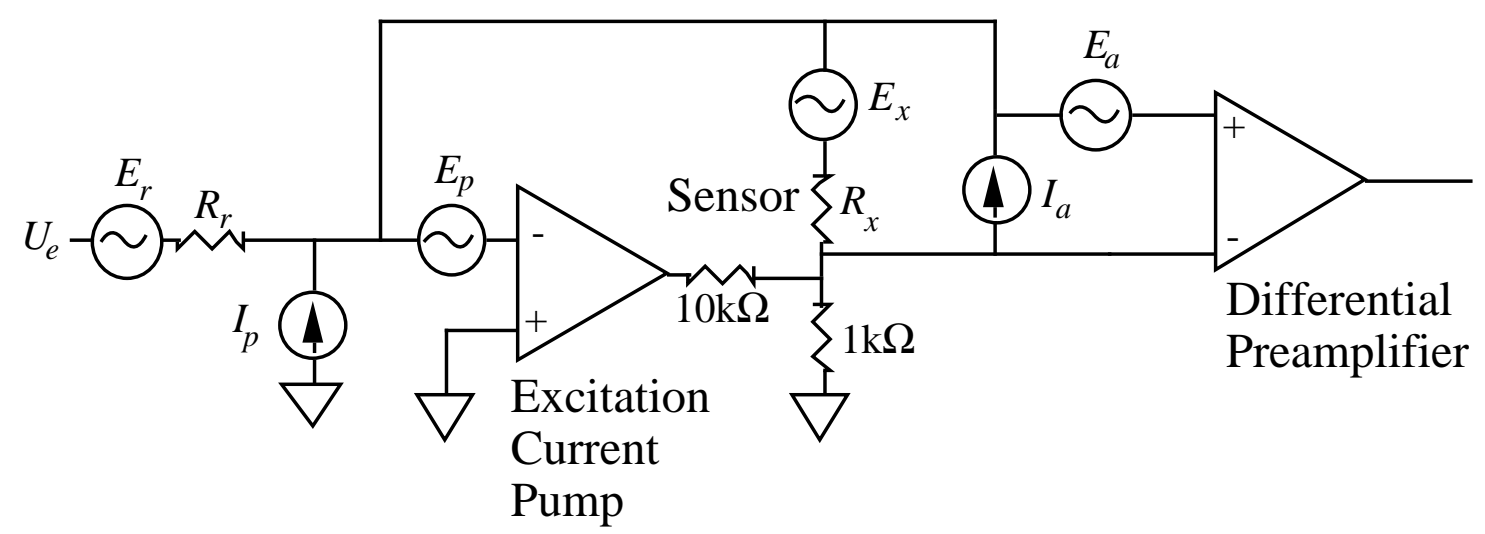

Figure 2: Input noise model for the self-balancing bridge of Figure 1. The amplifiers are modelled by ideal operational amplifiers with equivalent voltage and current noise sources. The noise due to the $10 \mathrm{k} \Omega$ and $1 \mathrm{k} \Omega$ voltage divider resistors is small in all conditions and is neglected.

The system noise performance limits the resolution of the resistance measurement at the lowest excitation. Figure 2 shows the noise model of the bridge circuit, which yields the total noise reduced to the input of the differential preamplifier

$$
E_{t o t}^{2}=\left(\frac{R_{x}}{R_{r}}\right)^{2}\left[4 k T R_{r}+E_{p}^{2}+R_{r}^{2} I_{p}^{2}\right]+4 k T_{x} R_{x}+R_{x}^{2} I_{a}^{2}+E_{a}^{2},
$$

where $E_{i}, I_{i}$ are the spectral densities of the noise voltages and currents, respectively, as shown in Figure 2. The first term on the right side of Eq. (3) is the noise due to the current pump, the second term is the thermal noise of the sensor resistor, and the last terms are due to the preamplifier. The thermal noise of the sensor is normally negligible if $T_{x}$ is low. After filtering due to the combination of the integrator and the inhibit switch, the squared output noise voltage is

$$
E_{n}^{2}=E_{t o t}^{2} \Delta B \frac{G^{2}}{1-2 f T_{\text {inhibit }}},
$$

where $\Delta B$ and $G$ are the system bandwidth and voltage gain, $f$ is the measurement frequency, and $T_{\text {inhibit }}$ is the duration of the inhibit gate signal. The model can be used for the noise optimisation of the components in the circuit.

Figure 3 shows the noise contributions of different sources together with the total noise at the highest resistance range which uses $R_{r}=100 \mathrm{k} \Omega$. At low resistances the preamplifier voltage noise dominates; the device uses the OP77 operational amplifiers with significant $1 / f$ contribution at $12.5 \mathrm{~Hz}$. At higher resistances the sensor noise becomes increasingly important, but the contribution of the current pump noise dominates at large resistances already before the midrange of the ADC. For lower resistance ranges the preamplifier and the excitation current 
pump noises dominate the RT sensor noise at low and high sensor resistance values, respectively.

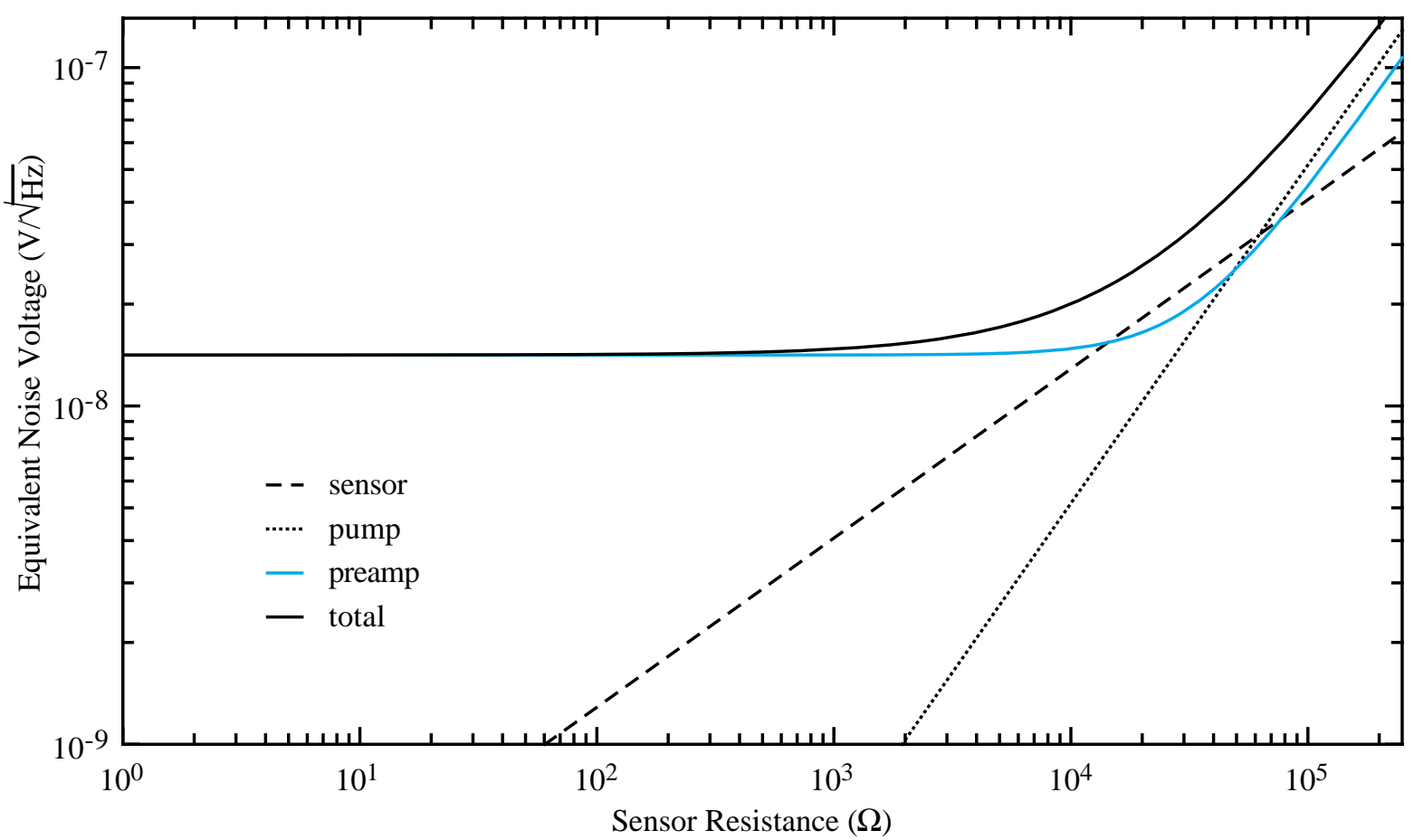

Figure 3: Theoretical contributions of different sources to the spectral density of the equivalent input noise voltage at $200 \mathrm{k} \Omega$ range and $1 \mathrm{nA}$ excitation current, with the sensor at room temperature.

The noise model of Eq. (3) yields the theoretical noise figure at $12.5 \mathrm{~Hz}$ shown in Figure 4, for the range of $200 \mathrm{k} \Omega$ and the lowest excitation current of $1 \mathrm{nA}$. We note that by improving the noise performance of the preamplifier and the current pump a significant gain can be expected. This is particularly important when the sensor is cold.

The output from the bridge circuit of each measurement unit is sampled with a 16-bit sigmadelta A/D converter at $10 \mathrm{~Hz}$ rate; there is thus one ADC in each channel which avoids multiplexing of the analogue signals. The design becomes therefore more simple and straightforward and the use of expensive special components such as fast mercury wetted relays can be avoided. The sampling speed can be kept high and it will not be limited by the circuit response speed.

The A/D conversion results are read at $10 \mathrm{~Hz}$ rate to a $\mathrm{PC}$ type computer using its parallel port. The computer interface is made extremely silent by using optoisolators which prevent parasitic signals, due to the fast digital circuits of the computer, from coupling to the sensor and the preamplifier. Several front-ends (in the current version eight) can be connected to one bus.

The mains power feed to the system power supply is RF filtered at the entrance of the EMI shielded enclosure of the circuitry. In our prototype version the lines of the parallel port of the $\mathrm{PC}$ are not optoisolated at the entrance of the enclosure, because in the final version an embedded PC will be installed inside. 


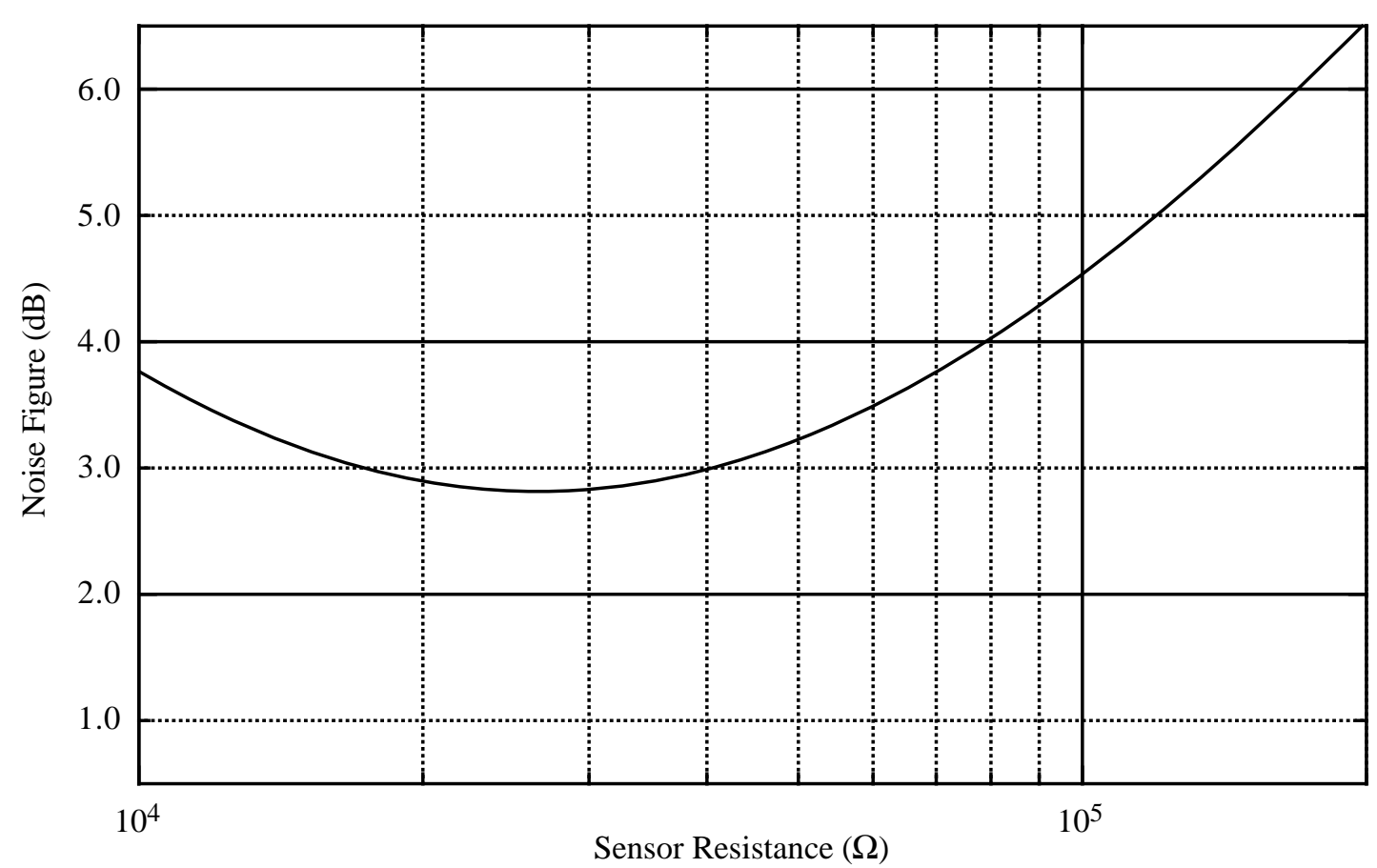

Figure 4: Theoretical noise figure of Eq. (3) for the self-balancing active bridge circuit model of Figure 2.

The leads connecting the sensors in the cryostat are RF filtered at the entrance of the metallic vacuum vessel of the cryostat, and the multiwire cable linking the cryostat to the EMI shielded enclosure is double shielded. This prevents the self-heating of the sensors due to RF signals, which is limiting the accuracy of resistance thermometry seriously below $100 \mathrm{mK}$ unless a shielded room is used for all equipment.

\section{USER INTERFACE AND COMMUNICATION WITH CONTROL SYSTEMS}

The system includes a dedicated control program in the PC which

- collects data at $10 \mathrm{~Hz}$ rate from all 8 channels,

- adds a time stamp to each reading,

- converts resistance values to absolute temperature,

- saves the data to a ring buffer,

- calculates the noise and the trend of the temperature records,

- performs digital filtering with desired filter shape,

- controls the excitation and resistance range settings,

- performs calibration and diagnostic self-tests,

- makes an alarm if user-defined operation limits are exceeded,

- communicates with a host computer which may request readout or new settings.

Alerts may be performed if the temperature is beyond limits or is changing too rapidly, for example. Digitally filtered temperature values and time stamps can be read by a host computer at the time desired, and the program includes customised modes of data acquisition to treat special situations such as magnet quenches differently from routine operation. 
Under normal conditions, old data stored in the ring buffer is continuously overwritten by new readings. In this way a flood of irrelevant information can be avoided, while there is still data available in the buffer in case something important happens so that the record of a thermal transient can be stored after a hardware or software trigger.

The control program communicates with a host computer using the TCP/IP protocol. This ensures the independence of the hardware realisation of the network and it can also tackle collisions in the case that several machines need the attention of a higher level unit simultaneously. This enables the construction of a large and easily scalable remotely controlled systems with a graphical user interface. We have built such an interface on a Macintosh computer using the LabVIEW graphical programming language "g".

The program may, for example, display the temperature data in graphical form resembling the strip of a chart recorder shown in Figure 5. In the example the system parameters can be changed simply by clicking the corresponding buttons in the interface panel, or by incrementing/decrementing the displayed numbers.

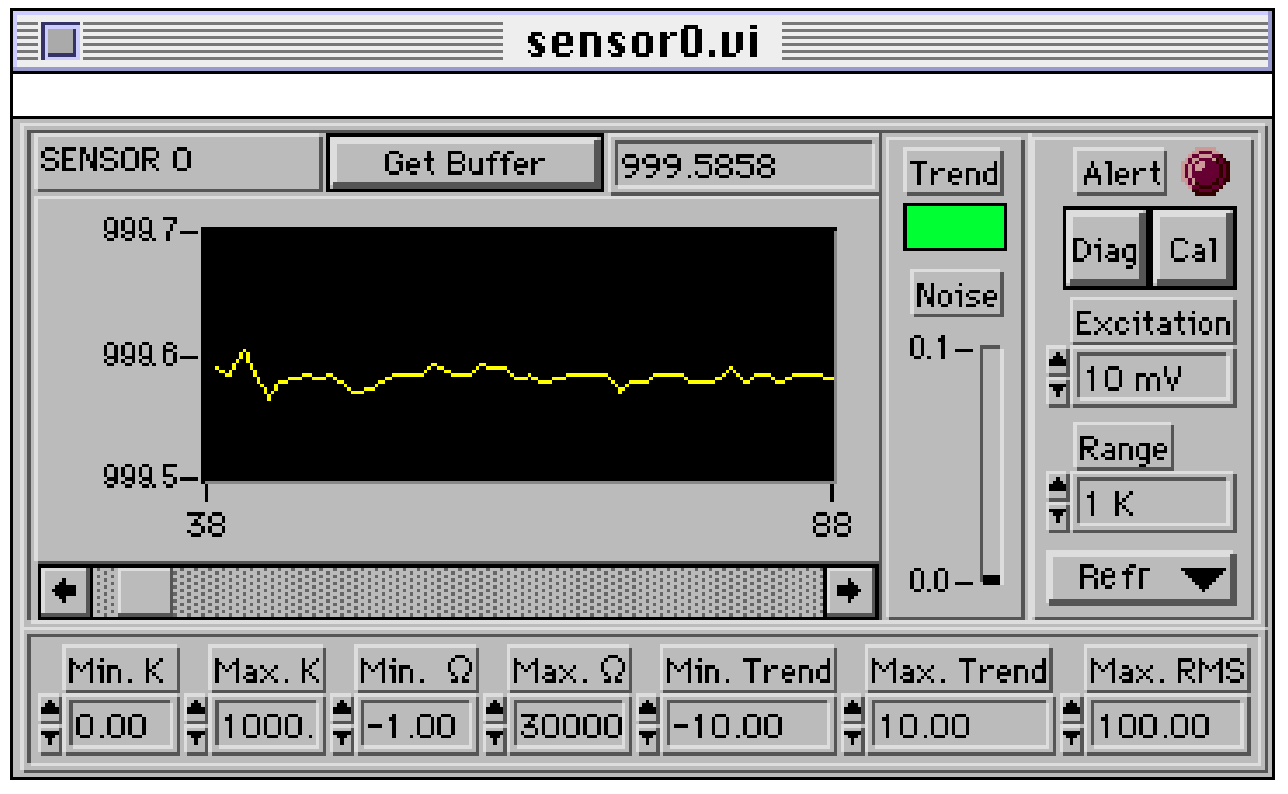

Figure 5: Graphical user interface showing the strip chart recording of one temperature sensor output. The value of the last averaged reading is also displayed numerically on the top, and a bar graph ("Noise") shows the RMS deviation from the average. The excitation and range settings are displayed on the right, and the alarm limits for the value, trend and noise are given on the bottom. A scroll bar allows to scroll the strip chart recording for old readings.

A logbook feature (Figure 6) is also included to keep track of the operations performed during a session and to help to relate their possible effects to the obtained data. The panel is also used to control the communication between the host and the PC, to set averaging or digital filtering parameters, to turn alerts on or off, and to begin and stop data acquisition. 


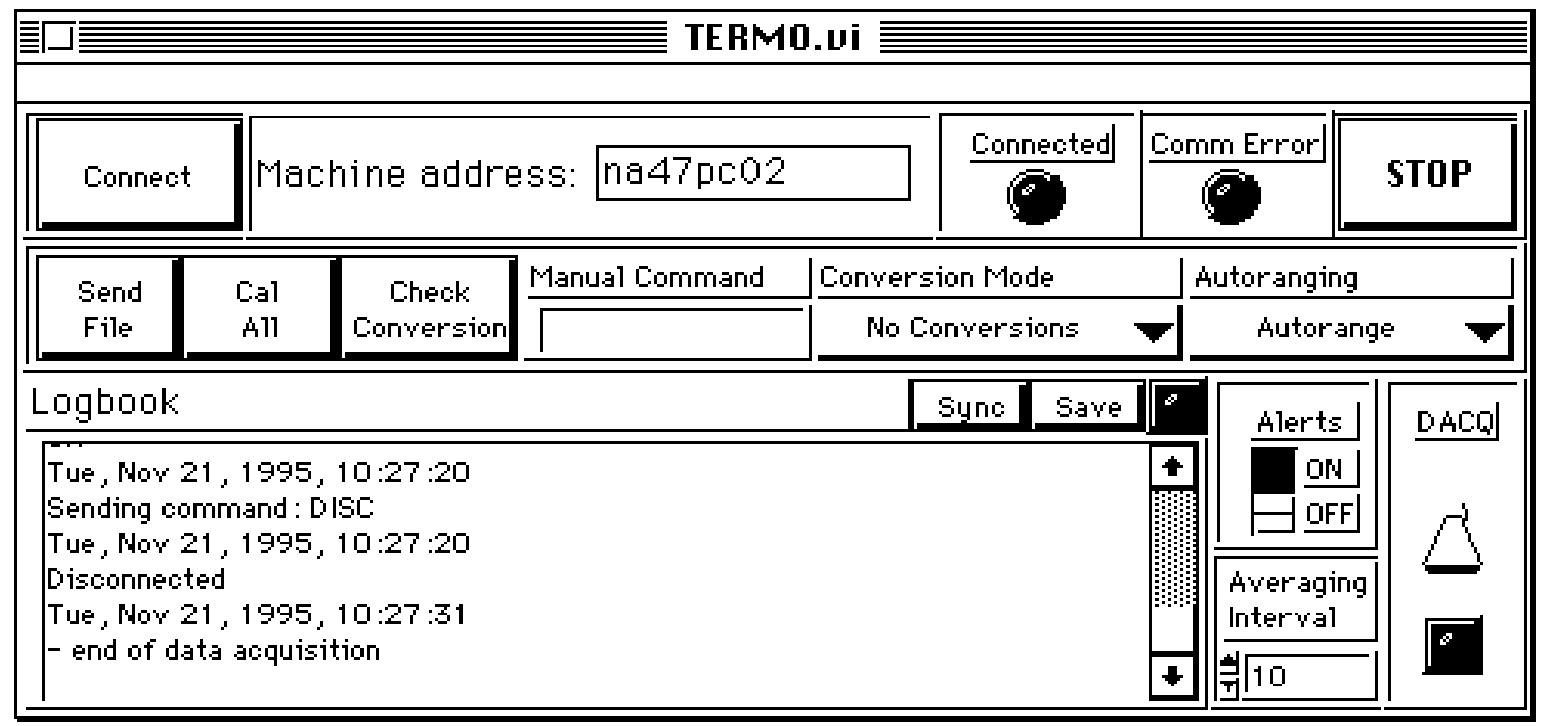

Figure 6: The main control panel with the logbook listing. The "Averaging interval" is the number of readings used for the calculation of the average, trend and RMS deviation of the temperature readings.

\section{PERFORMANCE}

The time-domain step response of the circuit at $2 \mathrm{k} \Omega$ range, at the three excitation settings, is given in Figure 7. The slower response predicted by Eq. (2) is clearly shown by the experimental data. The amplitude responses in the frequency domain were calculated from the experimental step responses; Figure 8 shows the comparison of the amplitude responses at 10 $\mathrm{mV}$ midrange excitation setting. The integral of the square of the experimental amplitude response was used as a noise bandwidth in the comparison of the measured noise with the spectral density of the noise model.

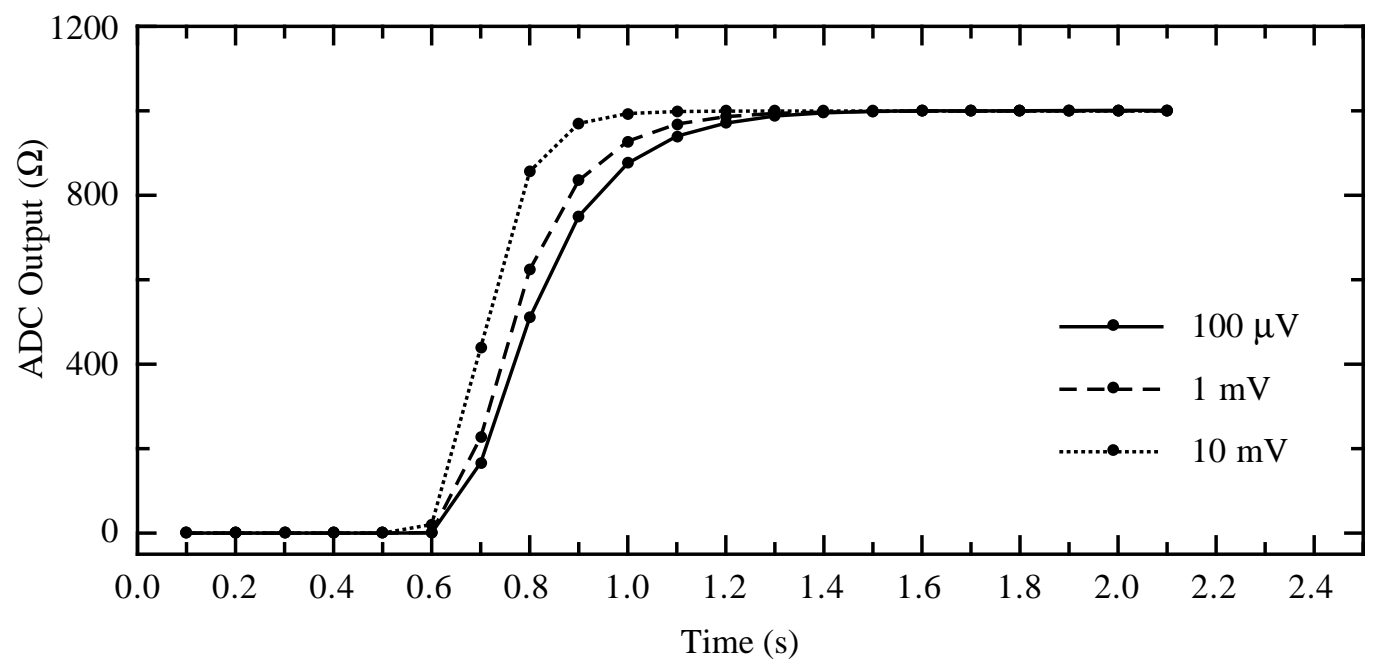

Figure 7: Response of the self-balancing active bridge circuit to a step change of the sensor resistance from 0 to $1 \mathrm{k} \Omega$. 


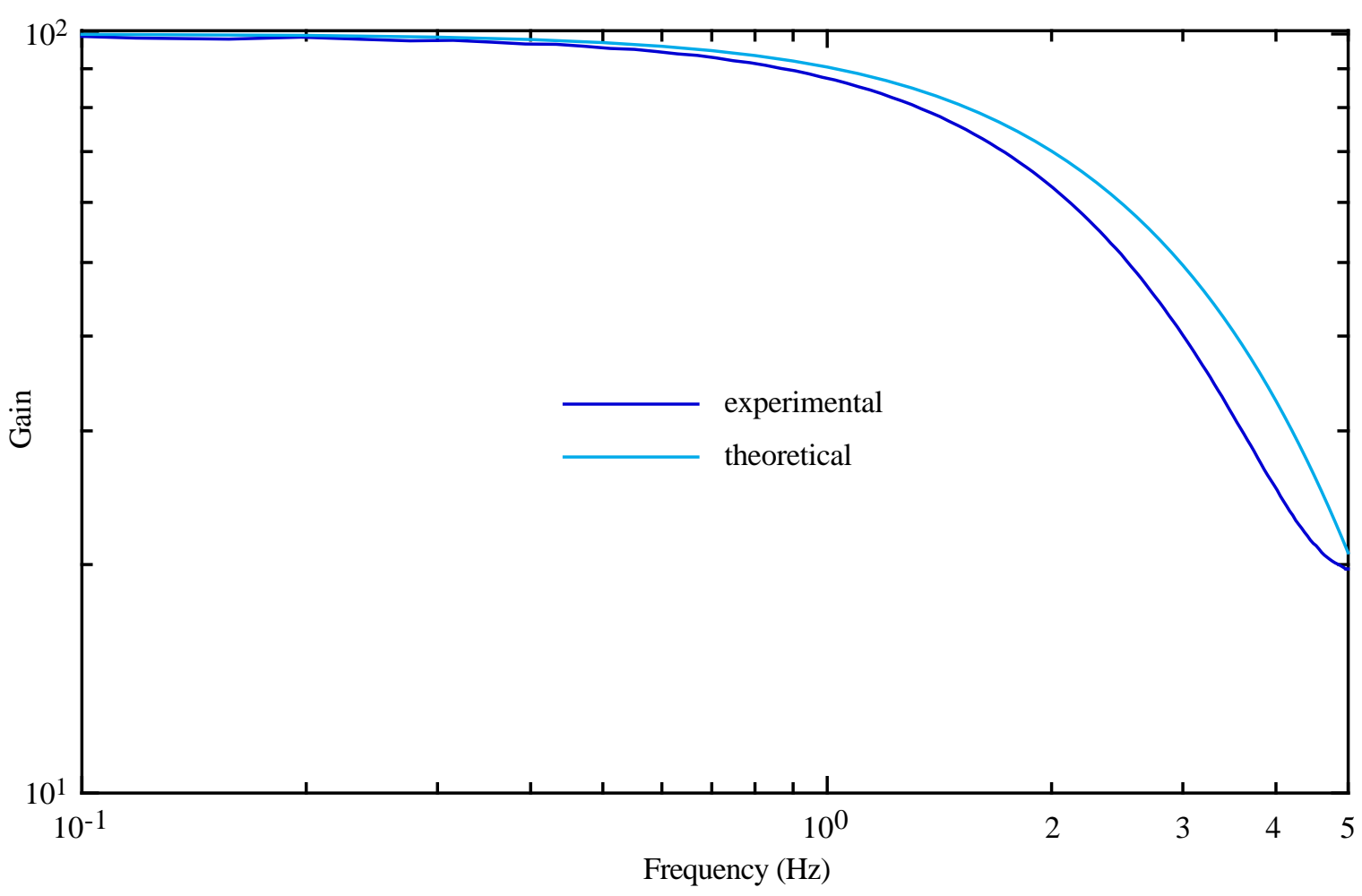

Figure 8: Frequency-domain amplitude response at $10 \mathrm{mV}$ midrange excitation setting and $1 \mathrm{k} \Omega$ sensor resistance. The experimental curve was determined from the time-domain step response of Figure 7.

The noise performance was evaluated from the experimental RMS resolution of the sensor resistance measured at the lowest excitation current $(100 \mu \mathrm{V}$ excitation voltage over the resistor at midrange) for all four resistance ranges. The test unit was the first prototype, which had $R_{r}=$ midrange sensor resistance, and OP77 operational amplifiers were used both in the current pump and in the preamplifier. In the noise bandwidth it is taken into account that two sidebands around the measurement frequency of $12.5 \mathrm{~Hz}$ contribute to the noise, but it is assumed that the noise is white, although we know that there is a significant $1 / f$-contribution in the OP77 operational amplifiers.

The circuit noise exceeds the quantization step of the 16-bit ADC only at the lowest excitation level. The resulting noise-dominated resolution of the circuit is shown in Figure 9 for all four ranges, together with the theoretical resolution calculated from the model of Eqs. (3) and (4) using the typical noise specifications of the operational amplifiers, and the theoretical response bandwidth, with the assumption of white noise around $12.5 \mathrm{~Hz}$. The theoretical curves agree with the measured points reasonably well, and the difference may be explained by some residual EMI, higher than typical noise of the amplifiers, and the contribution of the 1/f-noise.

The system stabilises thermally in 3 minutes after cold start. Changes in ambient temperature are reflected in the deviation of the measured resistance value by about $60 \mathrm{ppm} /{ }^{\circ} \mathrm{C}$ air temperature. 


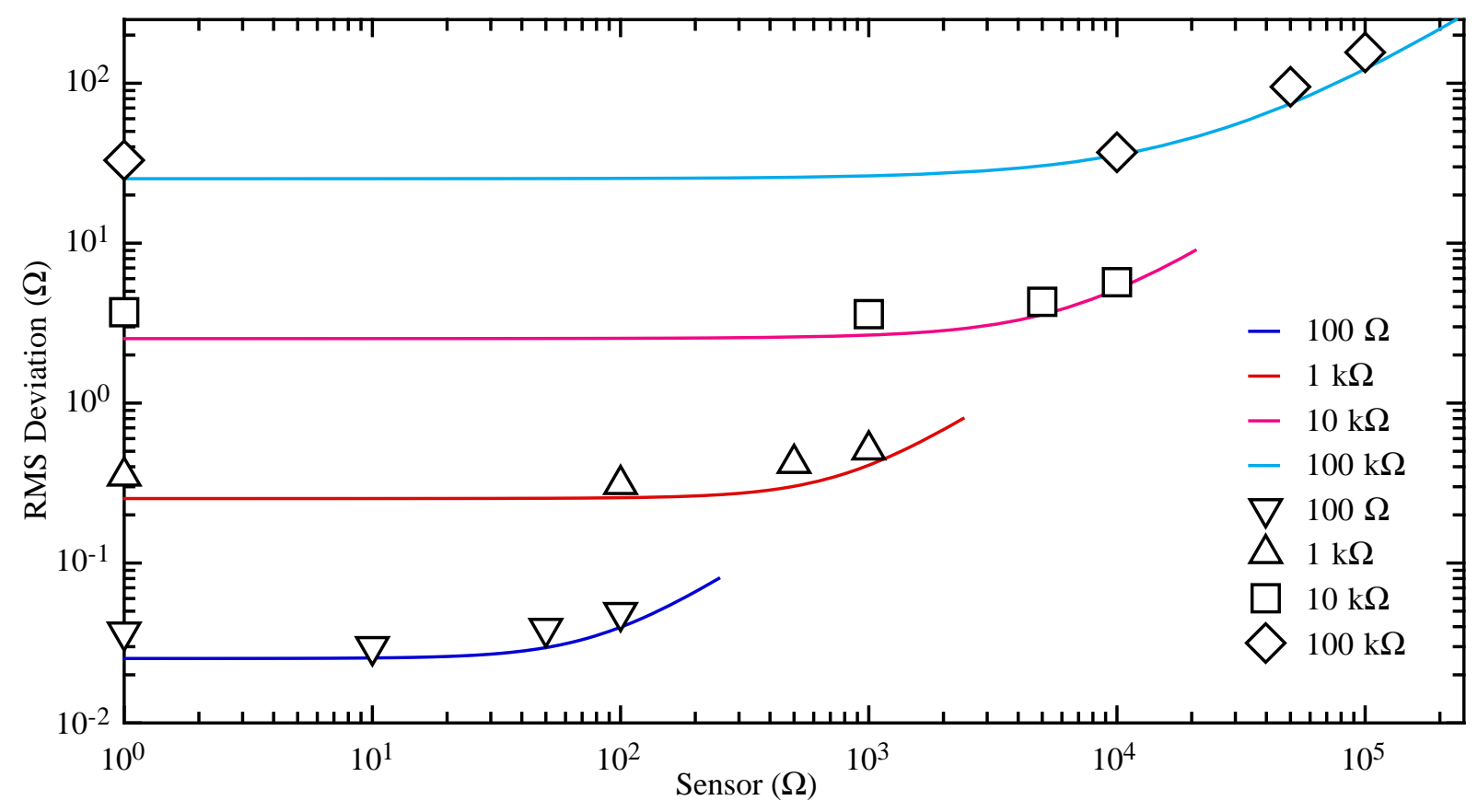

Figure 9: Comparison of the experimental and theoretical RMS deviation of the measured value of the sensor resistance for all the four measurement ranges, at the excitation current which gives the lowest $(100 \mu \mathrm{V})$ voltage over the sensor at midrange resistance values which are indicated in the labels of the theoretical curves and experimental data points.

The equipment has been used intensively in the CERN RD39 project [11] to control a continuous-flow helium cryostat and to measure the temperature dependence of the critical current in superconducting microstrip counters. Different types of sensors $(\mathrm{Ge}, \mathrm{Pt}$, carbon composite) have been used in the temperature range of $1.7 \ldots 300 \mathrm{~K}$. The resolution of temperature using a Ge sensor was $0.35 \mathrm{mK}$ at $1.8 \mathrm{~K}$ at the lowest excitation which causes 0.8 pW dissipation and negligible self-heating of the sensor. The temperatures of thermal shields at 20 and $50 \mathrm{~K}$ temperatures were monitored with $10 \mathrm{mK}$ resolution using Pt100 sensors and the two higher excitations, which at $50 \mathrm{~K}$ give 1 and $100 \mathrm{nW}$ loads, respectively. In this temperature range even the highest excitation produces a negligible self-heating if the sensor is mounted well. The recording of temperature dependent dynamic events such as quenches has been also tested experimentally in the same set-up.

\section{CONCLUSIONS}

We have produced and tested a prototype system for cryogenic resistance thermometry with features designed for facilitating the scaling-up in view of large applications of superconductivity. The circuit was modelled and tests indicate that the model is usable for the optimisation of the components for particular sensors if desired, as was discussed in Section 3.

Improvement in the noise performance can also be achieved without penalty in the cost, by the change of the operating frequency to $20 \mathrm{~Hz}$. This could be associated with a faster response of the circuit to step changes of the sensor resistance, so that the dynamic range of the circuit would be used better at the higher excitation currents. 
The projected frequency of $20 \mathrm{~Hz}$ would significantly reduce also the $1 / f$-noise. Elimination of the parasitics related with the mains power frequency of $50 \mathrm{~Hz}$ would require to decrease the integrator inhibit time to $5 \mathrm{~ms}$. Minor improvements in the EMI control are also possible as is suggested by the data points which are systematically above the theoretical lines in the Figure 9.

Other possible extensions of the present prototype system include a version with four measurement ranges from $20 \Omega$ to $20 \mathrm{k} \Omega$, and optimisation for the noise performance at low sensor resistance. Such a circuit would provide a resolution of $1 \mathrm{mK}$ with low-impedance sensors such as $\mathrm{Pt}$ and $\mathrm{RhFe}$ resistors, at temperatures above $15 \mathrm{~K}$.

A minor modification of the bridge circuit board allows the measurement of temperature using silicon diode sensors. In this case a constant noise-optimised $10 \mu \mathrm{A}$ DC excitation is used, and only one range is needed. Such a modified circuit was already tested out, with minor modifications of the control program adapting it to the changes in the self tests and control settings. The intelligent features in the control program also enabled the self-recognition of the card by the control program so that the current polarity and excitation settings are always automatically correct.

The prototype device uses a PC type computer as a processor. The same source code can be used without modification in an embedded PC which can be mounted inside the RF-tight enclosure.

\section{ACKNOWLEDGEMENT}

A special grant from the Finnish Ministry of Education for our project is gratefully acknowledged.

\section{REFERENCES}

[1] The LHC Study Group, The Large Hadron Collider - Conceptual Design, CERN Report CERN/AC/95-05 (LHC) (1995).

[2] M. Rabany, A Proposal to Move from the LEP Topological to an LHC Fuctional Control System Architecture, CERN LHC Note 365 CERN AT/95-55 (IC) (1995).

[3] O.V. Lounasmaa, Experimental Principles and Methods below $1 \mathrm{~K}$ (Academic Press, London 1974).

[4] H. Preston-Thomas, Metrologia 27 (1990) 3.

[5] L.G. Rubin, Cryogenics 10 (1970) 14.

[6] L.G. Rubin, B.L. Brandt and H.H. Sample, Cryogenics 22 (1982) 491.

[7] H.H. Sample, B.L. Brandt and L.G. Rubin, Rev. Sci. Instrum. 53 (1982) 1129.

[8] B.L. Brandt, L.G. Rubin and H.H. Sample, Rev. Sci. Instrum. 59 (1988) 642.

[9] A.C. Anderson, Rev. Sci. Instrum. 51 (1980) 1603.

[10] W.B. Bloem, Cryogenics 24 (1984) 159.

[11] J. Kyynäräinen, T. Niinikoski, Y. Semertzidis, R. Voss, P. Berglund, H. Seppä and I. Suni, Proposal DRDC P53: Superconducting Microstrip Detectors, CERN Proposal CERN/DRDC 93-53 (1993). 